\title{
Fire Fighting System Modeling in Oil and Gas Waterway Terminal Based on Colored Petri Nets
}

\section{Luiz Henrique Carneiro Valda ${ }^{1}$, Jandecy Cabral Leite ${ }^{2}$, Rivanildo D. Almeida ${ }^{3}$, João Paulo Apoliano Oliveira ${ }^{4}$}

\author{
1,3,4 Universidade Federal do Pará. Rua Augusto Corrêa, 01 - Guamá. CEP 66075-110 - Belém Pará - Brasil.PA. \\ ${ }^{2}$ Instituto de Tecnologia e Educação Galileo Da Amazônia (ITEGAM). Av.Joaquim Nabuco No 1950. Centro, Manaus-AM.
}

Email: luiz_valda@yahoo.com; jandecycabral@hotmail.com

\author{
Received: August 25 $5^{\text {th }}, 2017$ \\ Accepted: September 25th 2017 \\ Published: September 30th, 2017 \\ Copyright (C2016 by authors and Institute \\ of Technology Galileo of Amazon (ITEGAM). \\ This work is licensed under the Creative \\ Commons Attribution International \\ License (CC BY 4.0). \\ http://creativecommons.org/licenses/by/4.0/ \\ cc) (i) (2) Open Acces:
}

\begin{abstract}
Modeling, as a means of analysis of safety systems, as well as industrial processes and related purposes, meets the demand for suitable methods. The present work aims to evaluate an adherence of the concepts of modeling and Petri nets applied to the logic and interlock strategies of a Fire Fighting System (FFS). For this purpose, a real installation was used to acquire the information, which was an Oil \& Gas Waterway Terminal, located in the municipality of Coari in the State of Amazonas, Brazil, which receives, stores and disposes of oil and liquefied petroleum gas (LPG). The analysis models and the interlocking strategies are validated with the use of the Petri nets, where are the patterns of systems to discrete events, through a method of analysis in which all the paths and states that the modeled system are simulated It can obtain, to allow from this model to study and evaluate possibilities of improvement of the system.
\end{abstract}

Keywords: Modeling, Colored Petri Nets, Fire Fighting System, Waterway Oil Terminal.

\section{Modelagem de Sistema de Combate a Incêndio em Terminal Aquaviário de Petróleo e GLP Baseado em Redes de Petri Coloridas}

\begin{abstract}
RESUMO
A modelagem, como meio de análise de sistemas de segurança, bem como de processos industriais e finalidades afins, atende à demanda por métodos apropriados. $\mathrm{O}$ presente trabalho tem como objetivo avaliar a aderência dos conceitos de modelagem e de redes de Petri aplicados a lógica e estratégias de intertravamento de um Sistema de Combate a Incêndio (SCI). Para tal finalidade utilizou-se uma instalação real para a aquisição das informações, que foi um Terminal Aquaviário, situado no município de Coari no interior do Estado do Amazonas-Brasil, que recebe, armazena e escoa em navios, petróleo e gás liquefeito de petróleo (GLP). Os modelos das lógicas e estratégias de intertravamento serão validados com o uso de rede de Petri coloridas, onde são considerados os aspectos de sistemas a eventos discretos, por meio de um método de análise em que são simulados todos os caminhos e os estados que o sistema modelado pode atingir, permitindo que a partir desse modelo possam-se estudar possibilidades de melhoria do sistema.
\end{abstract}

Palavras Chaves: Modelagem, Redes de Petri Coloridas, Sistema de Combate a Incêndio, Terminal Petrolífero Aquaviário.

\section{INTRODUÇÃO}

As operações de transporte de hidrocarbonetos em terminais aquaviários envolvem o risco operacional intrínseco com potencial de incêndio e explosão, por este motivo operam dentro de limites vigentes em normas, padrões operacionais e de automação industrial, visando obter o melhor combate frente a situações de sinistros ou anomalias operacionais que envolvam os riscos supracitados.

O pronto combate é conseguido pela ação do sistema de combate a incêndio (SCI), alicerçado em planos e algoritmos de atuação e resposta, que devem ser executados pela equipe de 
brigada de emergência e controle operacional. $\mathrm{O}$ acontecimento de sinistros no transporte de óleo e gás é indesejado, e devido a isso, gerenciado. Entretanto, a equipe de controle operacional juntamente com o SCI deve estar apta a responder frente a qualquer situação de emergência.

Um dos mais relevantes sistemas que integram um terminal aquaviário que escoa petróleo e gás é o SCI, o seu funcionamento eficiente proporciona segurança as pessoas e ao patrimônio. A Associação Nacional de Proteção de Incêndio (National Fire Protection Association) [1] assevera que os principais requisitos de um sistema de combate a incêndio no desenvolvimento do projeto de uma planta industrial são: (a) garantir a integridade física humana; (b) proteger o patrimônio e (c) permitir a continuidade das operações.

Os requisitos do SCI devem, de um modo abrangente, seguir um conjunto de regras e normas que delimitam os itens mínimos e os contornos do sistema. Estas normas são constituídas por instituiçõos reguladoras, como a Associação Brasileira de Normas Técnicas no Brasil (ABNT) e a NFPA norte americana. Entretanto, as normas contemporâneas consideram apenas as situações convencionais, onde o SCI opera de modo independente a outros sistemas industriais.

Por conta das características inovadoras das táticas que abrangem a eficaz integração de sistemas, estas não são necessariamente exploradas pelas normas e regramentos usuais supracitados, porém não quer dizer que elas não autorizem novas soluções. Táticas de controle unificadas têm sido promovidas [1], onde é sugerido o uso de estratégias complementares aos habituais e se admite a relevância de atividades de pesquisa nessa área de conhecimento.

Com fulcro na importância que o SCI possui nas indústrias em geral, e em especial na área de terminais aquaviário de transporte de óleo e gás, é notório que seus requisitos e operação precisem de metodologias de modelagem e análise, que busquem encontrar as melhores características de projeto e requisitos de operação visando salvaguardar a segurança das pessoas, meio ambiente e do patrimônio da unidade.

Com a assistência de sistemas automatizados, é possível desenvolver um SCI para garantir a segurança tanto da estrutura física e patrimônio quanto das pessoas dentro e arredores das instalações protegidas. Deste modo, devido à complexidade do SCI, torna-se premente uma modelagem que tenha aderência aos requisitos e torne possível analisar a dinâmica do sistema bem como sua integração com outros sistemas industriais, isto corrobora para o estudo e desenvolvimento de metodologias para auxiliar e guiar o desenvolvimento de modelos.

Desta maneira, um modelo é compreendido como uma abstração e representação simples do sistema [2]. A modelagem demonstra os componentes mais relevantes do sistema e o modo como eles se integram. Após de obtido o modelo do sistema, é possível executar a simulação com fins de se fazer a análise da dinâmica do sistema ou para análise de novas táticas de controle [3].

Simulações usam de modelos para caracterizar o funcionamento de um sistema que pode ou não existir. $O$ entendimento crucial é que a simulação é um desenvolvimento alternativo que se aproxima do sistema real e, em diversos aspectos, a finalidade da simulação é analisar e compreender a dinâmica deste em função de transações e disparos alternativos [3].

A modelagem de SCI, abrange a consonância do conhecimento de como esses sistemas operam. Ou seja, quais as tarefas realizadas por seus componentes e como são as interrelações entre estas ações e disparos. Nesta forma, é notório que os itens que compõem o SCI têm suas reações dinâmicas definidas em função da mudança de estados discretos como consequência do acontecimento de eventos instantâneos e que compõem um tipo de sistemas chamado de Sistemas a Eventos Discretos (SEDs) [4][5].

Em realidade, abordagens acadêmicas e de estudos sobre a dinâmica do fogo, da chama, da fumaça, das pessoas em situações de sinistro [6], etc., asseveram que conceitos de sistemas híbridos e difusos são também relevantes na análise de SCI. O sistema híbrido é composto por apresentar ao mesmo tempo valores com características contínuas e/ou com características discretas [7]. Porém, em um enfoque holístico visando as funcionalidades dos itens de um SCI, o ensejo deste como um SED é adequado considerando que o seu objetivo está em variáveis e valores específicos (discretos) que podem até ser contínuas, ou seja, os estados e funcionalidades do SCI são projetados em função de uma checagem feita por comparação simples de valores, como por exemplo: menor que, igual a, maior que, etc. Utilizando o critério das Redes de Petri é exeqüível analisar a funcionalidade dos processos realizados pelo SCI.

Com fulcro em [8], a rede de Petri se caracteriza como uma das mais poderosas ferramentas gráficas utilizadas para a modelagem, análise e projeto de SEDs. Esta técnica admite a representação clara e compilada das funções e do desenvolvimento dos processos, proporcionando a caracterização da dinâmica do sistema, bem como a realização das táticas de controle. A rede de Petri é ideal para a caracterização de sistemas com processos concorrentes, não sincronizados, paralelos e distribuídos [9-11].

Desta maneira, várias abordagens decorrentes de rede de Petri foram propostas com corroborada eficiência para a análise, modelagem e controle de exemplos reais de SEDs. Estas metodologias são utilizadas por meio de um enfoque estrutural, onde o sistema é modelado em distintas camadas de abstração. Como exemplo, tem-se o caso de sistemas de automação industrial, onde tem sido usado um modelo teórico, obtido através do uso da metodologia Esquema do Fluxo de Produção (Production Flow Schema - PFS) [4] e a compilação do supracitado sistema para modelos funcionais por meio do uso de Grafo de Marco de Fluxo (Mark FlowGraph - MFG) [12] e suas vertentes extras, onde são mantidas as estruturas das "ações" caracterizadas na camada superior da modelagem. Os modelos obtidos podem, portanto, ser analisados e validados por simulação conforme os requisitos das atividades e serviços de um sistema de automação industrial e checado de acordo com as características da rede de Petri [13].

\section{REVISÃO BIBLIOGRÁFICA}

\section{II.1. O PARADIGMA DAS REDES DE PETRI}

Diferentes linguagens formais podem ser escolhidas para modelar um dado sistema a eventos discretos como de combate a incêndio. Por exemplo, podem ser consideradas redes de filas, máquinas de estados finitos ou autômatos, redes de Petri ou processos semi-Markov generalizados [14].

Nesse contexto, o paradigma das redes de Petri foi selecionado por várias razões. Em primeiro lugar, uma vantagem indiscutível das redes de Petri é a representação gráfica e matricial. Esta característica particular das redes de Petri a torna muito intuitiva e poderosa para a análise estruturada, bem como para a avaliação de desempenho e simulação.

Uma segunda característica interessante das redes de Petri é sua maneira natural de representar comportamentos complexos que incluem paralelismo e sincronização [15], e para a quantidade de conhecimento nos estudos acerca das redes de Petri que foi desenvolvido nas últimas décadas [16].

Em qualquer problema de decisão, o sistema em questão deve apresentar um ou mais graus de liberdade, permitindo escolher diferentes configurações alternativas como resultado de 
uma decisão. Esses graus de liberdade ou características indefinidas do sistema de eventos discretos original podem ser expressos no modelo resultante por meio de parâmetros indefinidos, levando também ao conceito de rede de Petri indefinida [17].

Os parâmetros de uma rede de Petri podem desempenhar papéis diferentes e, posteriormente, podem ser classificados de acordo com diferentes tipos, tais como parâmetros estruturais, de marcação, de transição ou de interpretação. Os parâmetros estruturais estão relacionados à estrutura do modelo de rede de Petri, enquanto os demais tipos estão associados ao seu comportamento.

Uma visão geral das diferentes abordagens para resolver um problema de otimização com base em uma rede de Petri indefinida pode ser encontrada em Ref. [18][19].

\section{MATERIAIS E MÉTODOS}

A descrição e os modelos foram desenvolvidos a partir do levantamento de documentação e dados técnicos e teóricos oriundos do Sistema de Gerenciamento Eletrônico de Documentos Corporativos da companhia proprietária das instalações e de dados práticos coletados no Terminal Aquaviário de Coari, no Município de Coari, Amazonas.

Se comparadas com outras técnicas de descrição de Sistemas de Combate a Incêndio, as redes de Petri [20][10] possui um poder maior de modelagem, além disso ela tem a característica e vantagem da facilidade de visualização do sistema. Para a construção dos modelos em rede de Petri pode-se utilizar a técnica do PFS (production flow schema) [4], que é uma classe de rede de Petri devidamente interpretada para sistemas produtivos. O PFS baseia-se em aplicar refinamentos sucessivos através de uma abordagem "top-down", de maneira que seja possível inserir progressivamente no modelo o detalhamento dos processos do sistema. O objetivo é representar claramente o fluxo de operações tendo como referência a evolução dinâmica de um determinado processo. A partir do PFS pode ser gerado um outro modelo em rede de Petri para descrever o comportamento dinâmico detalhado do sistema.

A modelagem e a análise das propriedades dos modelos, com o apoio da equipe de manutenção do TA-Norte, foram validadas no editor CPN-Tools, desenvolvido pela, da Universidade de Aarhus, Dinamarca, apartir de 2010 CPN Tools foi transferido para o grupo AIS da Universidade de Tecnologia de Eindhoven, Holanda.

\section{III.1. APRESENTAÇÃO INFORMAL DAS REDES DE PETRI COLORIDAS}

Uma pertinente limitação das redes de Petri ordinárias é o fato de necessitar uma grande quantidade de lugares e de transições para representar sistemas complexos (também nomeados pela literatura como sistemas reais). Ao passo em que a rede cresce, a visão geral do sistema modelado, por parte do analista, começa a ser dificultada ou comprometida e a análise do sistema modelado, por parte do computador, começa a ser restringida ou também dificultada.

Os sistemas reais corriqueiramente apresentam processos similares que ocorrem de modo paralelo ou concorrente, diferindo entre si apenas pelos seus recursos ou produtos manipulados. As fichas, por expressarem basicamente se as condições associadas a um lugar são verdadeiras ou falsas, requerem uma grande quantidade de lugares e, consequentemente, de transições, para representar tais sistemas com processos ou disposições similares.
Uma opção neste contexto é ao invés de aumentara quantidade de lugares, diferenciar os processos, recursos ou produtos através das fichas. Pois quando as fichas, ao invés de conter apenas função binária, contenham um vetor de dados ou informações, a quantidade de lugares e transições de uma rede que modela um sistema real pode ser reduzida.

Esta é o paradigma das redes de Petri coloridas, tema tratado neste capítulo. Em seguida, será realizada uma abordagem informal do tema supracitado, mostrando suas vantagens e vulnerabilidades se comparado às redes de Petri lugar-transição. Logo após, é elencado um exemplo, oriundo do trabalho de [21], que ilustra a evolução do conceito das redes ordinárias para o conceito das RPC.

Considerando hipoteticamente que existem dois tipos de processos em um sistema qualquer, denominados processos-p e processos-q, que compartilham três tipos de recursos, denotados por recursos-r, recursos-s e recursos-t. Tais processos ocorrem paralelamente e demandam os insumos citados ao mesmo tempo.

Os processos-p podem ser separados em quatro estados principais. $\mathrm{O}$ primeiro estado indica a quantidade de processos- $\mathrm{p}$ a serem operados. O segundo estado necessita da utilização de dois recursos-s para a execução do sistema. O terceiro estado requer a utilização de dois recursos-s e um recurso-t para a execução do sistema. Por fim, o quarto estado necessita utilizar dois recursos-s e um recurso-t para a execução do sistema.

No que se refere aos atos mais relevantes dos processosp, é possível sobressair a primeira ação, que utiliza dois recursos-s para que o sistema siga do estado inicial para o segundo estado, a segunda e a terceira ação, que buscam um recurso-t para que o sistema siga do segundo para o terceiro estado e do terceiro para o quarto estado e, por fim, a quarta ação, que reinicia o processo-p.

Os processos-q podem ser separados em cinco principais estados. O primeiro estado indica a quantidade de processos-q a serem operados. O segundo estado necessita da utilização de um recurso-r e de um recurso-s para a operação do sistema. O terceiro estado requer a utilização de um recurso-r e de dois recursos-s para a operação do sistema. O quarto estado necessita da utilização de dois recursos-s para a operação do sistema. Por fim, o quinto estado demanda a utilização de dois recursos-s e um recurso-t para a operação do sistema.

No que se refere aos atos mais significativos dos processos-q, é possível sobressair a primeira ação, que utiliza um recurso-r e um recurso-s para que o sistema siga do estado inicial para o segundo estado, a segunda ação, que utiliza um recurso-s para o sistema siga do segundo para o terceiro estado, a terceira ação, que libera um recurso-r para que o sistema siga do terceiro para o quarto estado, a quarta ação, demanda um recurso-t para que o sistema siga do quarto para o quinto estado e, por fim, a quinta ação, que reinicia o processo-q.

Na Figura 1 é ilustrada a modelagem do sistema descrito anteriormente. Os lugares Bp. Cp, Dp e Ep se referem respectivamente aos estados 1, 2, 3 e 4 dos processos-p. As transições T2p, T3p, T4p e T5p se referem respectivamente aos eventos 1, 2, 3 e 4 dos processos-p. Consideração semelhante pode ser aplicada aos lugares e transições que estão no lado direito da rede de Petri ilustrada, que se referem, aos estados e ações dos processos-q descritos anteriormente. Os lugares $\mathrm{R}, \mathrm{S}$ e $\mathrm{T}$ correspondem aos recursos-r, recursos-s e recursos-t, respectivamente.

Na RdP ilustrada na Figura 1, faz-se premente notar os dois tipos de processos por duas sub-redes separadas, ainda que os dois processos distintos compartilhem os mesmos recursos, utilizando-os de uma forma análoga ou semelhante. 


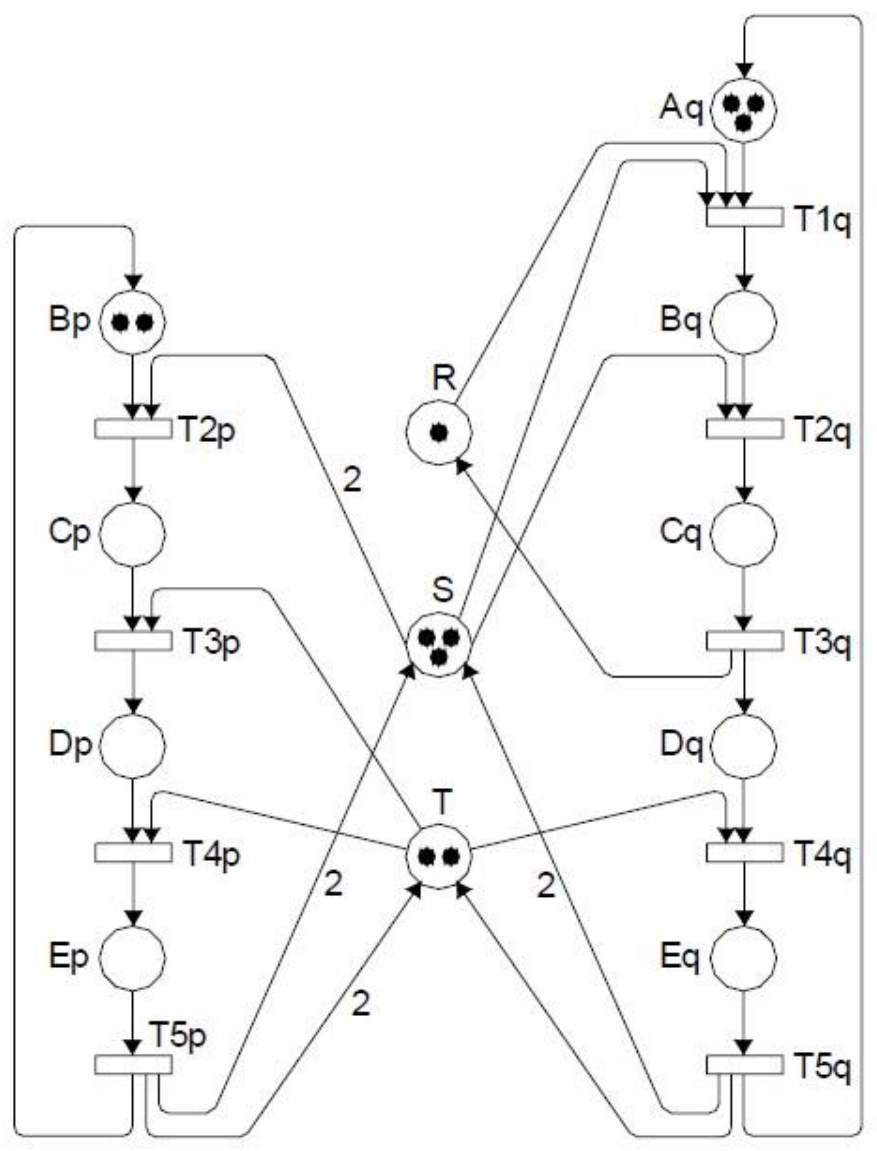

Figura 1: RdP descrevendo a alocação de recursos do sistema (marcação inicial M0).

Fonte: [21].

No que diz respeito à visão do analista sobre o sistema modelado, a rede ilustrada torna possível a visualização e sensibilização das restrições operacionais do sistema. Essa vantagem se esvai ou diminui a medida que o sistema modelado se torna maior ou mais complexo.

No que tange à implementação do modelo, a utilização de sub-redes similares causa por conseqüência ineficiência computacional, demonstrada pela entrada e pelo processamento dos dados. Tal deficiência é tolerável ou aceitável para um sistema simples, como o representado na Figura 1. Entretanto, para sistemas maiores, redundâncias como estas podem acarretar em um custo computacional elevado ou proibitivo.

Sistemas de manufatura, de telecomunicações ou de automação industrial, usualmente, apresentam diversos processos com características de paralelismo ou concorrência, compartilhando muitos recursos. Considerando hipoteticamente que o sistema a ser modelado, ao invés de contar com dois tipos de processos e três insumos distintos, apresentasse dezenas de processos e recursos. A representação gráfica do sistema e sua análise seria dificultada e pouco inteligível e a implementação computacional do modelo, assim como seu processamento, seriam bastante restritivos ou complexos.

Uma opção tática para suprimir esse tipo de problema é aplicar o conceito das redes de Petri hierárquicas. Neste tipo de rede, considera-se a existência de sub-redes que se comportam como lugares ou como transições e, deste modo, uma RdP passa a incluir mais dois conjuntos de elementos: os macro-lugares, que representam uma sub-rede por um lugar, e as macro-transições, que representam uma sub-rede por uma transição.

Embora teoricamente interessantes e potencialmente úteis, as redes de Petri hierárquicas não resolvem eficientemente o problema do crescimento excessivo do número de nós da rede, quando se pretende modelar um sistema não trivial, com fulcro em [22]. Essa é, portanto, uma das grandes limitações das redes de Petri lugar-transição na modelagem e análise de sistemas reais.

Assevera que uma representação mais compacta de uma rede de Petri é obtida através da incorporação, para cada ficha, de uma cadeia de dados, chamados cores da ficha. O conceito de cor é análogo ao conceito de tipo da variável, comum nas linguagens de programação [21].

Na Figura 2 é ilustrado o mesmo sistema apresentado na Figura 1, porém modelado utilizando as redes de Petri coloridas.

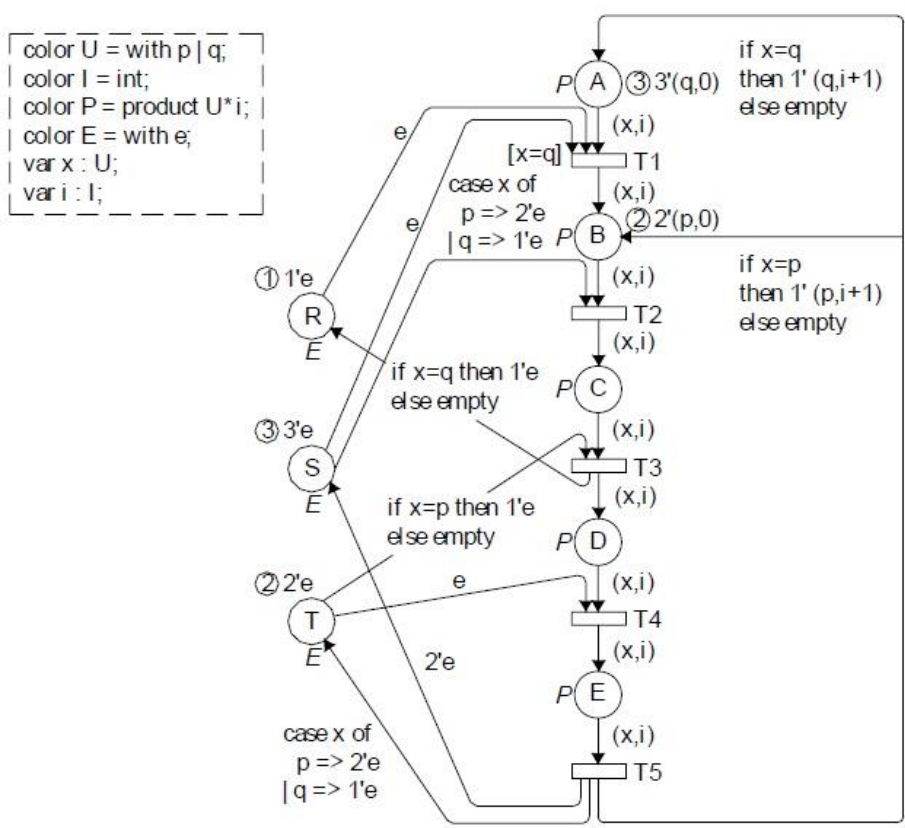

Figura 2: RPC descrevendo a alocação de recursos do sistema (marcação inicial M0).

Fonte: [21].

Para um determinado lugar $\mathrm{P}$, as fichas nele contidas devem possuir cores que correspondam a um determinado tipo. Este tipo é denotado conjunto de cores do lugar. Examinando a rede ilustrada na Figura 2, no lugar R, todas as fichas nele contidas serão do tipo E. Assim é possível notar que a abstração de cores é usado nas redes de Petri coloridas de modo análogo a abstração dos tipos de variáveis está para as linguagens de programação.

Para os arcos são agregadas variáveis, expressões matemáticas ou elementos de uma linguagem de programação. Para se efetuar o disparo de uma transição, as estruturas de dados contidas nas fichas devem satisfazer as condicionais contidas nos arcos da rede de Petri colorida, de modo semelhante como interagem as fichas e os pesos dos arcos em uma rede de Petri ordinária.

No canto superior à esquerda da Figura 2, é possível notar a existência de um retângulo feito por linhas tracejadas, contendo declarações de uma linguagem de programação. Qualquer linguagem pode ser utilizada em modelos de RPC, entretanto, no contexto contemporâneo é vastamente utilizado, tornando-se quase uma regra, a utilização do padrão ML, que possui uma sintaxe textual semelhante à da linguagem de programação $\mathrm{C}$.

$\mathrm{O}$ criador das redes de Petri coloridas, impulsionou pesquisas dentro deste paradigma de modelagem. O Departamento de Ciência da Computação da Universidade de Aarhus tornou-se a maior referência mundial no tema em questão e desenvolveu a ferramenta computacional mais robusta já desenvolvida, até a atualidade, para a edição e análise de RPC: o CPN Tools. O CPN Tools utiliza uma linguagem similar ao padrão ML, chamada de CPN ML [22]. 
A Expressão $[\mathrm{x}=\mathrm{q}]$, associada à transição $\mathrm{T} 1$ da rede ilustrada na Figura 2, é classificada como uma guarda. Uma guarda é uma regra que delimita o disparo de uma transição apenas a uma variável de um tipo especificado. No exemplo em questão, a guarda $[\mathrm{x}=\mathrm{q}]$ especifica que a transição $\mathrm{T} 1$ só está habilitada para processos do tipo q, não efetuando disparos para processos do tipo p ou qualquer outro.

\section{III.2. APRESENTAÇÃO FORMAL DAS REDES DE PETRI COLORIDAS}

O entendimento, por parte do analista, da lógica matemática que rege uma RPC não é uma condição indispensável para a utilização desta técnica para a modelagem de um sistema. Com fulcro em [22], é possível especificar qualquer rede apenas em termos da sua representação gráfica.

A definição formal, entretanto, permite ao analista um maior detalhamento e compreensão da técnica utilizada, além de permitir analisar propriedades e atributos do modelo. Ao se aprofundar nos estudos acerca das redes de Petri coloridas, a compreensão da sua definição formal torna-se premente.

Asseveram que uma rede de Petri colorida é um grafo bipartido dirigido representado por uma quíntupla [23]:

$$
\mathrm{CPN}=(P, T, C, I, O),
$$

onde:

- P é um conjunto finito de lugares de dimensão n;

- T é um conjunto finito de transições de dimensão m;

- C são os conjuntos de cores associados com os lugares e com as transições de modo que:

$$
\begin{aligned}
& \mathrm{C}\left(p_{i}\right)=\left\{\mathrm{a}_{\mathrm{i} 1}, \ldots, \mathrm{a}_{\mathrm{iui}}\right\}, \mathrm{u}_{\mathrm{i}}=\left|\mathrm{C}\left(p_{i}\right)\right|, \mathrm{i}=1, \ldots, \mathrm{n} . \\
& \mathrm{C}\left(\mathrm{t}_{\mathrm{j}}\right)=\left\{\mathrm{b}_{\mathrm{j} 1}, \ldots, \mathrm{b}_{\mathrm{jvj}}\right\}, \mathrm{v}_{\mathrm{i}}=\left|\mathrm{C}\left(t_{j}\right)\right|, \mathrm{j}=1, \ldots, \mathrm{m} .
\end{aligned}
$$

Os símbolos a's e b's representam as cores associadas.

- I é uma aplicação de entrada $\mathrm{C}(p) \times \mathrm{C}(t) \rightarrow \mathrm{N}$ (inteiros não-negativos) correspondendo ao conjunto de cores direcionadas dos arcos de $P$ para $T$.

- $O$ é uma aplicação de saída $\mathrm{C}(t) \times \mathrm{C}(p) \rightarrow \mathrm{N}$ (inteiros não-negativos) correspondendo ao conjunto de cores direcionadas dos arcos de $\mathrm{T}$ para $\mathrm{P}$.

$$
\text { É possível notar que } \mathrm{O}(p, t)=\mathrm{O}^{\mathrm{T}}(t, p) \text {. }
$$

A junção de dados às fichas torna o modelo mais compacto, por outro lado aumenta a complexidade das funções de disparo. Nas RdP ordinárias, são associados aos arcos de entrada I e de saída. Os valores inteiros, enquanto que nas RPC, são associadas aos arcos funções complexas.

\section{RESULTADOS E DISCUSSÕES}

\section{IV.1. DESENVOLVIMENTO DO MODELO}

O paradigma mais abrangente da modelagem de redes de Petri coloridas a ser aplicada em algum sistema de combate a incêndio de um terminal aquaviário petrolífero, será baseado no apontamento dos estados, que serão desempenhados pelos lugares, dos eventos que serão desempenhados pelas transições, e das informações complementares do modelo, a serem representadas pelas cores (tipos de dados).
Após a criação, desenvolvimento e a análise do modelo, é possível avaliar as opções táticas de melhoria disponíveis. É premente salientar que todo SCI de um TA possui suas particularidades, sendo necessário adaptar o modelo a cada cenário analisado. No caso das operações de SCI em terminais aquaviários, temos:

\section{(i) Lugares}

Os lugares de uma rede Petri colorida devem representar estados como: (a) quantidade de hidrocarbonetos armazenado nas tancagens do TA; (b) equipamentos disponíveis para uso, como bombas de incêndio, sprinklers, válvula de alimentação do canhão monitor ou dispersor de espuma; (c) sensores ativados; e (d) ações dos operadores da unidade ao pressionar botoeiras de emergência.

\section{(ii) Transições}

As transições de uma rede Petri colorida devem representar eventos tais como: (a) ações de partida, desligamento e abertura e fechamento canhões monitores e aspersores; e (b) retorno dos equipamentos ao repouso após o fim do sinistro.

\section{(iii) Cores}

As cores associadas às fichas deverão armazenar informações como por exemplo: (a) identificação do tipo de sinistro, se com petróleo ou GLP e em que local ocorre, em um tanque ou esfera; e (b) atributos dos equipamentos, se a bomba esta disponível ou em manutenção, horímetro da bomba, nível de operação dos tanques ou esferas, e se a válvula e canhões estão abertos ou fechados.

\section{IV.2. DESCRIÇÃO DA LÓGICA OPERACIONAL DO SISTEMA DE COMBATE A INCÊNDIO DO TERMINAL}

Com base em visitas de reconhecimento e documentação técnica, é possível descrever a lógica operacional do sistema a ser modelado, que consiste no conjunto de procedimentos a serem realizados para as operações. A lógica e filosofia operacional do controle automático do sistema de combate a incêndio do TA Coari, no que se refere às operações do sistema de combate a incêndio, pode ser descrita do seguinte modo a seguir.

A lógica do sistema consiste em fornecer ao parque de tancagem de dispositivos automáticos que combatam o incêndio na área e a disponibilizar uma rede de água de combate a incêndio permanentemente monitorada com relação à pressão e vazão requeridas.

O suprimento de água à rede de combate a incêndio é proveniente do rio Solimões e a captação é feita diretamente do rio, pelas duas bombas da rede de combate a incêndio.

O Terminal dispõe ainda de um castelo de água conectado à rede de combate a incêndio, mantendo-a sempre pressurizada em cerca de $1,0 \mathrm{kgf} / \mathrm{cm}^{2}$, pela altura estática da água.

A rede fica permanentemente pressurizada por uma linha de água potável ligada à uma caixa d'água elevada. Esta linha tem uma chave de fluxo de vazão alta (FSH). A abertura de qualquer sistema da rede provocará uma vazão alta na linha de água potável e a chave de vazão fará partir a bomba e acionará uma chave de pressão temporizada (PSL) na rede de água.

Se depois de um tempo determinado, suficiente para entrada em regime da bomba, a pressão estiver baixa, a chave acionará a partida da segunda bomba, em caso de falha na bomba, detectado pelo sensoriamento interno da bomba, é gerado um sinal 
de "falha bomba" que causa que a respectiva bomba que emitiu o sinal entre automaticamente em manutenção, após seu reparo e/ou avaliação a mesma recebe sinal de "bomba ok", que torna a bomba novamente disponível para a operação, o modelo em redes de Petri colorida da lógica de partida das bombas, tolerante a falhas, é mostrado na Figura 3, após partida a bomba é obedecido o detalhamento lógico do sistema para cada área do terminal onde o SCI atua.

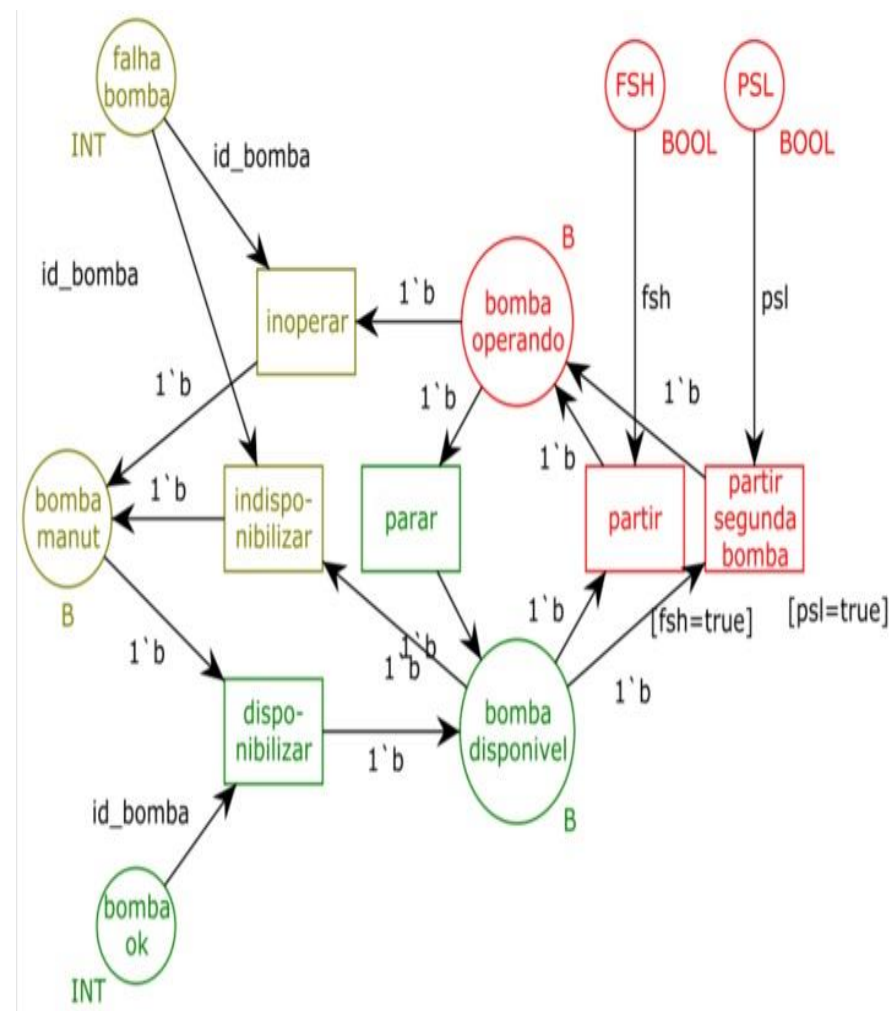

Figura 3: Modelagem da lógica de partida das bombas do SCI. Fonte: Autores, (2017).

\section{IV.3. DESCRIÇÃO DA OPERAÇÃO DO SCI}

A seguir, é descrita a operação do SCI do Terminal Aquaviário de Coari, no que se refere ao tratamento de uma situação de sinistro.

O sinistro pode ser acionado remotamente ou localmente por um operador, ou acionado automaticamente pelo PLC de automação industrial da unidade a partir do desenvolvimento de uma lógica de intertravamentos, sendo acionado o sinistro o mesmo será analisado quanto a que em que local da planta industrial ocorre e qual produto está envolvido, deste modo é partida a bomba e simultaneamente é iniciada a mobilização dos ativos de SCI pertinentes a área afetada, que notadamente podem ser as área de tancagem de petróleo ou área de esferas de GLP.

$\mathrm{Na}$ área de tancagem, em caso de sinistro em TQ-02/03, serão acionadas por intertravamentos as válvulas de entrada de água para resfriamento dos costados do tanque sinistrado e do vizinho, em caso de sinistro em TQ-01/04, serão acionadas por intertravamentos as válvulas de entrada de água para resfriamento dos costados do tanque sinistrado e também do seu vizinho TQ-04 (Diesel), a modelagem em redes de Petri colorida do intertravamento do subsistema do SCI que abrange a área de tancagem de petróleo é mostrada na Figura 4.

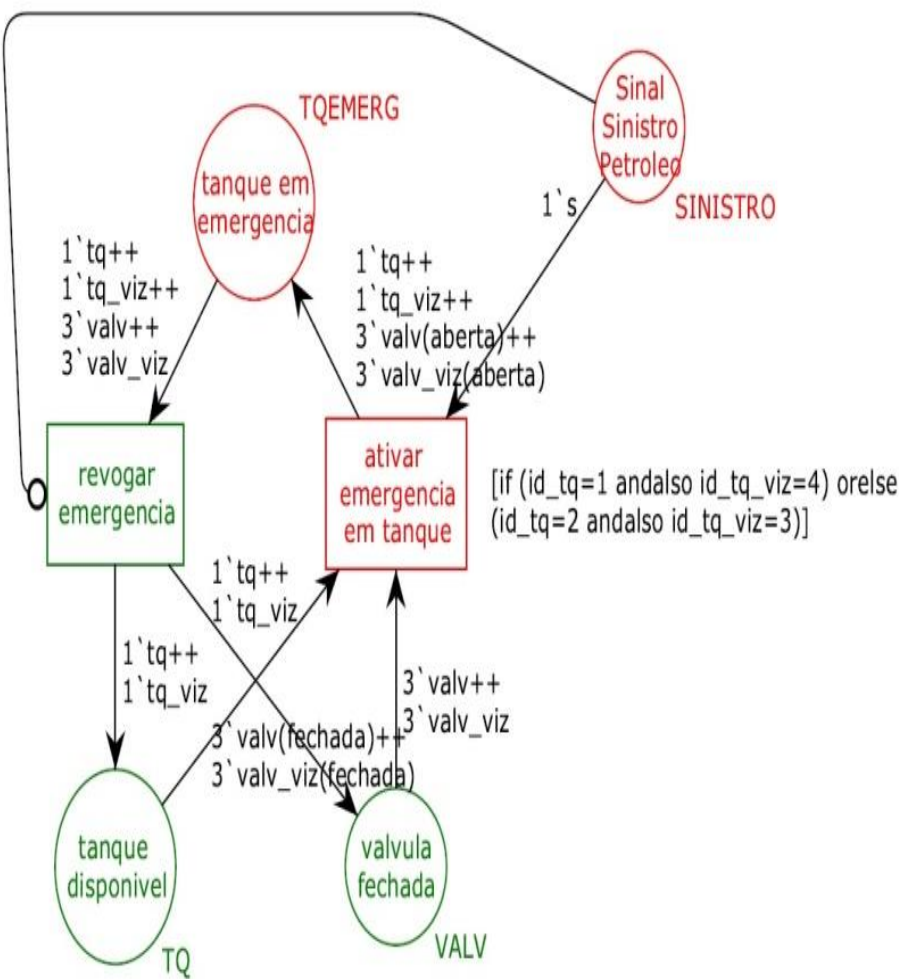

Figura 4: Modelagem em RPC da lógica do SCI na área de tancagem de petróleo.

Fonte: Autores, (2017).

$\mathrm{Na}$ área das esferas, em caso de sinistro em alguma das esferas EF-8001/8002/8003/8004/8005 e 8006, serão acionadas por intertravamentos os respectivos aspersores e o par de canhões monitores correspondentes. Devido à distância entre os costados das esferas serem de $35 \mathrm{~m}$ ou maiores, e conforme item 5.5 .3 da norma N-1203 [24], só é necessário resfriar esferas cuja distância entre o costado for menor de $30 \mathrm{~m}$ em relação a uma esfera sinistrada, não serão acionados aspersores ou canhões de esferas vizinhas a uma esfera sinistrada, a modelagem em redes de Petri colorida do intertravamento do subsistema do SCI que engloba a área das esferas é ilustrada na Figura 5.

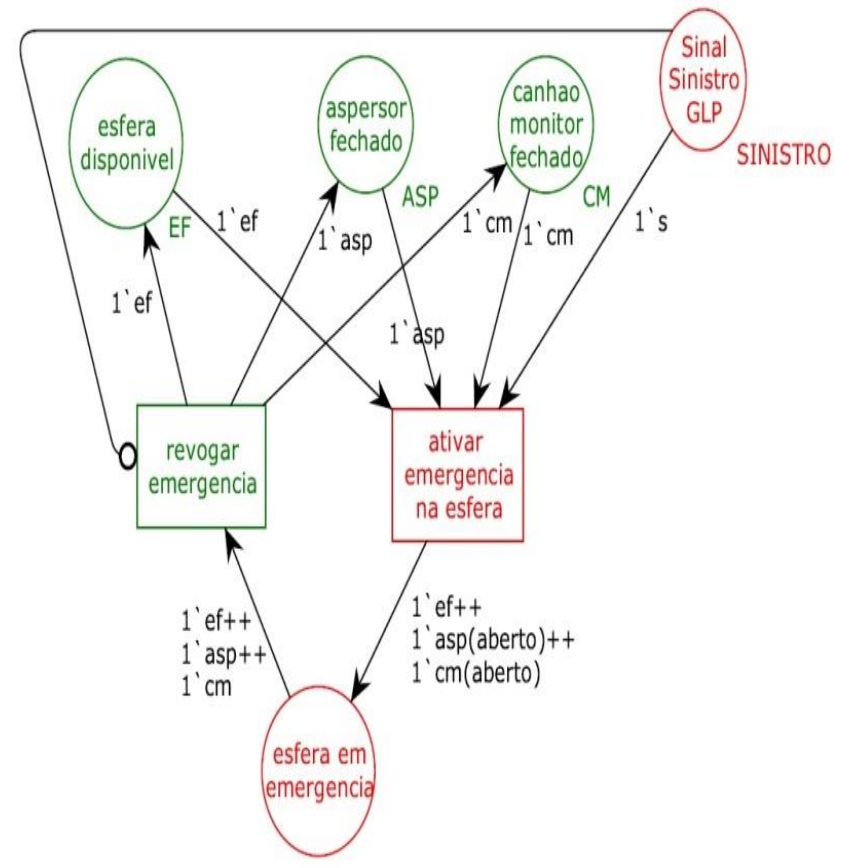

EF_EMERG

Figura 5: Modelagem em RPC da lógica do SCI na área das esferas de GLP.

Fonte: Autores, (2017). 


\section{IV.4. SISTEMA MODELADO}

Levando em consideração o exposto no item 4.1, assim Figura 6. terminal no tópico 4.2 e 4.3 , desenvolveu-se um modelo matemático, baseado em redes de Petri coloridas geral para o SCI,

\section{SINISTRO}

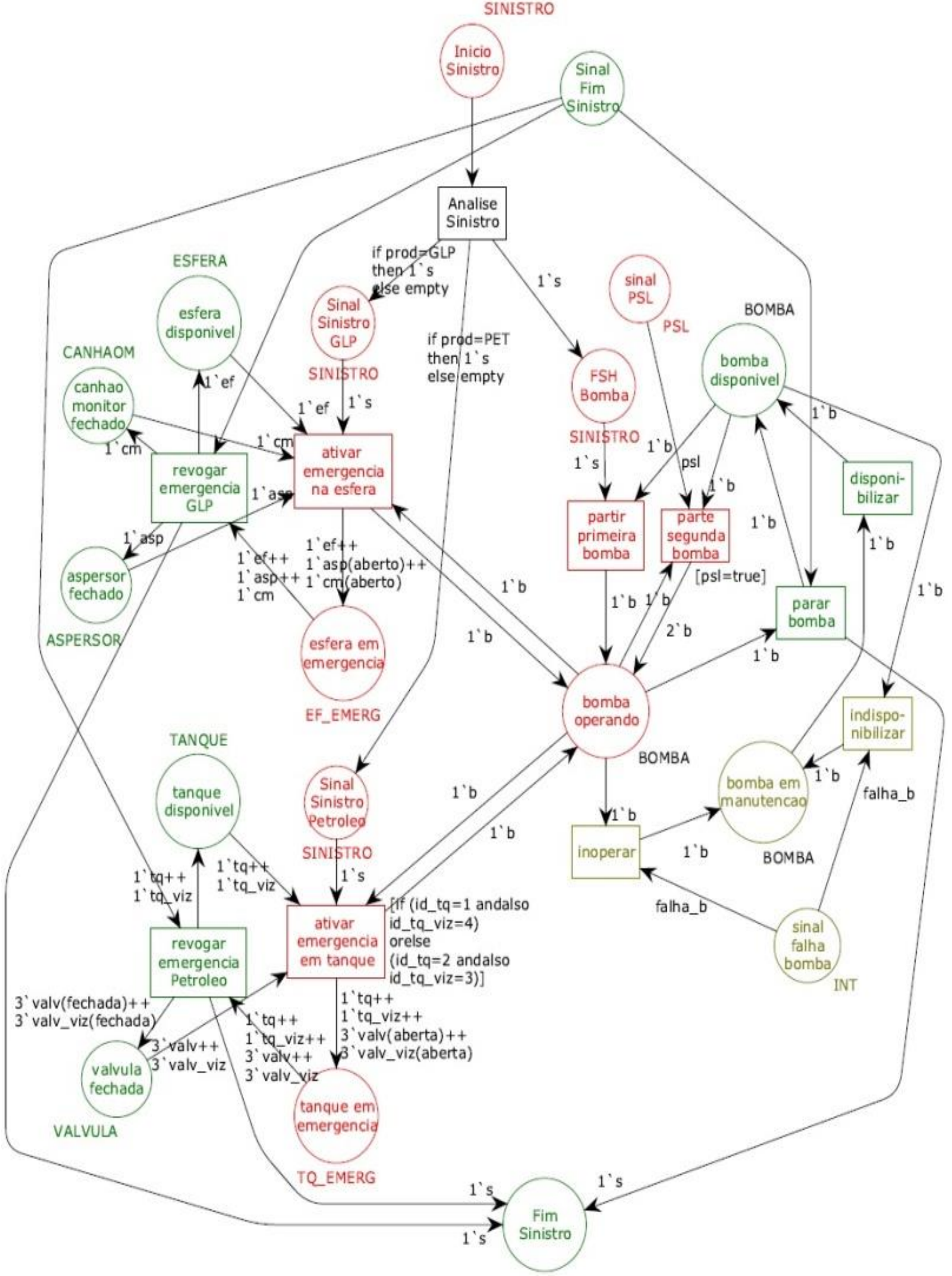

Figura 6: Operação do SCI do Terminal Aquaviário de Coari por uma rede de Petri colorida.

Fonte: Autores, (2017). 
Nas Tabelas 1, 2, 3 e 4 são descritos, respectivamente, os lugares, as transições, as cores e as variáveis do modelo proposto.

Tabela 1: Legenda dos lugares da rede apresentada na Figura 6.

\begin{tabular}{|c|c|}
\hline Lugar & Descrição \\
\hline Inicio sinistro & $\begin{array}{l}\text { Sinal de início de sinistro, gerado por operador ou } \\
\text { PLC. }\end{array}$ \\
\hline Sinal Fim $s$ & Sinal de fim de sinistro, gerado por operador. \\
\hline FSH Bomba & $\begin{array}{l}\text { Após início de um sinistro, vazão alta FSH na rede de } \\
\text { incêndio. }\end{array}$ \\
\hline Bomb & Bombas que estão disponíveis para operação. \\
\hline Bomba operando & Bombas que estão em operação devido a um sinistro. \\
\hline $\begin{array}{l}\text { Bomba em } \\
\text { manutenção }\end{array}$ & Bombas que estão em manutenção. \\
\hline Sinal $\mathrm{f}$ & $\begin{array}{l}\text { Sinal de falha da bomba, gerado por seus sensores } \\
\text { internos. }\end{array}$ \\
\hline Sinal PSL & Pressão baixa na rede de incêndio. \\
\hline Sinal s & Sistema detecta que sinistro ocorre na área do GLP. \\
\hline Esfera d & Esferas que estão disponíveis para operação. \\
\hline \begin{tabular}{|c|}
$\begin{array}{c}\text { Canhao monitor } \\
\text { fechado }\end{array}$ \\
\end{tabular} & Canhões monitores que protegem as esferas fechados. \\
\hline Aspersor fechado & Aspersores que protegem as esferas fechados. \\
\hline $\begin{array}{c}\text { Esfera em } \\
\text { emergência }\end{array}$ & $\begin{array}{l}\text { Esfera em emergência, juntamente com aspersor e } \\
\text { canhões monitores respectivos abertos. }\end{array}$ \\
\hline $\begin{array}{l}\text { Sinal sinistro } \\
\text { Petroleo }\end{array}$ & Sistema detecta que sinistro ocorre na área do Petróleo \\
\hline Tanque disponível & Tanques que estão disponíveis para operação. \\
\hline Válvula fechada & $\begin{array}{l}\text { Válvulas, que alimentam protetores dos tanques, } \\
\text { fechadas. }\end{array}$ \\
\hline $\begin{array}{l}\text { Tanque em } \\
\text { emergência }\end{array}$ & $\begin{array}{l}\text { Tanque em emergência, juntamente com as válvulas } \\
\text { respectivas abertas. }\end{array}$ \\
\hline $\mathrm{Fi}$ & $\begin{array}{l}\text { SCI e todos seus equipamentos estão desmobilizados } \\
\text { em todas as áreas. }\end{array}$ \\
\hline
\end{tabular}

Fonte: Autores, (2017).

Tabela 2: Legenda das transições da rede apresentada na Figura 6.

\begin{tabular}{|c|l|}
\hline Transição & \multicolumn{1}{|c|}{ Descrição } \\
\hline Análise Sinistro & Sistema analisando sinal de sinistro. \\
\hline $\begin{array}{c}\text { Ativar emergência } \\
\text { na esfera }\end{array}$ & $\begin{array}{l}\text { Mobilizando recursos do SCI referentes da esfera } \\
\text { sinistrada. }\end{array}$ \\
\hline $\begin{array}{c}\text { Revogar } \\
\text { emergência GLP }\end{array}$ & $\begin{array}{l}\text { Desmobilizar recursos do SCI referentes a esfera } \\
\text { sinistrada. }\end{array}$ \\
\hline $\begin{array}{c}\text { Partir primeira } \\
\text { bomba }\end{array}$ & $\begin{array}{l}\text { Primeira bomba é ligada, após sinal de vazão alta } \\
\text { (FSH). }\end{array}$ \\
\hline $\begin{array}{c}\text { Parte segunda } \\
\text { bomba }\end{array}$ & $\begin{array}{l}\text { Segunda bomba é ligada, após sinal de pressão baixa } \\
\text { na rede do SCI (PSL). }\end{array}$ \\
\hline $\begin{array}{c}\text { Parar bomba } \\
\text { Para a bomba após o fim do sinistro. }\end{array}$ \\
\hline $\begin{array}{c}\text { Inoperar Bomba } \\
\text { Indisponibilizar } \\
\text { bomba }\end{array}$ & Bomba sai de operação por falha. \\
\hline $\begin{array}{c}\text { Disponibilizar } \\
\text { bomba }\end{array}$ & Bomba fica indisponível por falha. \\
\hline $\begin{array}{c}\text { Ativar emergência } \\
\text { em tanque }\end{array}$ & $\begin{array}{l}\text { Mobilizando recursos do SCI referentes ao tanque } \\
\text { sinistrado. }\end{array}$ \\
\hline $\begin{array}{c}\text { Revogar } \\
\text { emergência }\end{array}$ & $\begin{array}{l}\text { Desmobilizar recursos do SCI referentes ao tanque } \\
\text { sinistrado. }\end{array}$ \\
\hline Fonte: Os autores (2017). \\
\hline
\end{tabular}

Tabela 3: Descrição das cores da rede apresentada na Figura 6.

\begin{tabular}{|c|c|}
\hline Cor & Descrição \\
\hline SINISTRO & Conjunto de cores relacionado ao sinistro. \\
\hline BOMBA & Conjunto de cores relacionado a bomba. \\
\hline PSL & $\begin{array}{l}\text { Conjunto de cores relacionado ao sensor de pressão } \\
\text { baixa PSL. }\end{array}$ \\
\hline INT & Conjunto de cores relacionado as falhas da bomba. \\
\hline ESFERA & Conjunto de cores relacionado a esfera. \\
\hline CANHAOM & $\begin{array}{l}\text { Conjunto de cores relacionado aos canhões } \\
\text { monitores das esferas. }\end{array}$ \\
\hline ASPERSOR & $\begin{array}{l}\text { Conjunto de cores relacionado aos aspersores das } \\
\text { esferas. }\end{array}$ \\
\hline EF_EMERG & 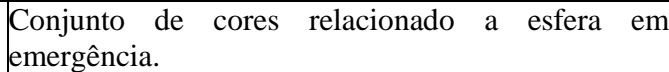 \\
\hline TANQUE & Conjunto de cores relacionado ao tanque. \\
\hline VALVULA & $\begin{array}{l}\text { Conjunto de cores relacionado as válvulas do } \\
\text { tanque. }\end{array}$ \\
\hline TQ_EMERG & $\begin{array}{l}\text { Conjunto de cores relacionado ao tanque em } \\
\text { emergência. }\end{array}$ \\
\hline
\end{tabular}

Fonte: Autores, (2017).

Tabela 4: Descrição das variáveis da rede apresentada na Figura 6.

\begin{tabular}{|c|c|l|}
\hline Variável & Tipo & \multicolumn{1}{|c|}{ Descrição } \\
\hline s & SINISTRO & $\begin{array}{l}\text { Identificação do sinistro com local e } \\
\text { produto. }\end{array}$ \\
\hline b & BOMBA & Bomba com tag, horimetro e status. \\
\hline falha_b & INT & $\begin{array}{l}\text { Falha na bomba identificada por número } \\
\text { inteiro. }\end{array}$ \\
\hline psl & PSL & Evento de pressão baixa na rede de SCI. \\
\hline ef & ESFERA & Esfera com tag, nível e status. \\
\hline cm & CANHAOM & Canhão monitor com tag e status. \\
\hline asp & ASPERSOR & Aspersor com tag e status. \\
\hline tq & TANQUE & Tanque com tag, nível e status. \\
\hline valv & VALVULA & Válvula com tag e status. \\
\hline
\end{tabular}

Fonte: Autores, (2017).

O modelo desenvolvido objetiva representar a dinâmica operacional do funcionamento e movimentação tática do SCI do TA Coari no advento de um sinistro. O incremento de cores busca aumentar a semelhança do modelo como instrumento refletor da realidade do sistema de emergência, pois corrobora para a diferenciação dos diferentes cenários e lógicas de intertravamento em função das características do sinistro no terminal supracitado.

Como se reconheceu a dinâmica da operação do SCI do TA como aderente com a teoria dos Sistemas a Eventos Discretos (SED), os lugares representam os estados do sistema e as transições os eventos que implicam na mudança de estados.

Os conjuntos de cores associados aos lugares especificam as variáveis que podem ser associadas a cada lugar. Por exemplo, no lugar EF_EMERG, só podem ser armazenadas as variáveis "ef", "cm" e "asp". 
A variável "s" caracteriza um evento de sinistro. Os números e dados associados a essa variável pode ser relacionado com um banco de dados que descreva minuciosamente as peculiaridades mais significativas do tipo de sinistro como, por exemplo, tipo de produto, local, condições de contorno da área afetada.

A variável "b" caracteriza uma bomba de combate a incêndio. Os dados associados a essa variável podem ser da identificação da bomba, seu horímetro e seu status. Assim como a variável "falha_b" caracteriza um evento de falha em determinada bomba. A variável "psl” caracteriza o advento de pressão baixa da rede do SCI, detectado por um sensor do tipo pressostato.

A variável "ef" caracteriza cada esfera. Os dados associados a essa variável pode ser relacionado com uma base de dados que descreva minuciosamente as peculiaridades mais significativas do equipamento como, por exemplo, características, identificação, nível operacional e status do equipamento.

A variável "cm" caracteriza cada canhão monitor e a variável "asp" caracteriza cada aspersor montados em uma esfera. Deve-se proceder analogamente ao que foi proposto para a variável "tq" para tanques de petróleo e "valv" para válvulas de SCI montados no tanque.

Essa diferenciação dos componentes do sistema traz como vantagens: i) poder avaliar, individualmente, a eficiência de cada equipamento empregado na operação do SCI do terminal; e ii) simular e aferir o tempo em que um sinistro é atendido em determinado cenário.

\section{CONCLUSÃO}

O presente trabalho derivou de uma pesquisa cuja finalidade era o desenvolvimento de um modelo de avaliação de desempenho operacional de SCI em terminais aquaviários que movimentam hidrocarbonetos, enfocando as operações de sinistro em tanques e esferas de armazenamento. Considerando que um TA pode ser considerado como um Sistema a Eventos Discretos, o modelo desenvolvido foi baseado em redes de Petri coloridas, sendo aplicado ao caso do Terminal Aquaviário de Coari, situado no Estado do Amazonas.

A metodologia, apresentada neste trabalho, para avaliação de desempenho operacional de SCI em TAs, mostrou-se de grande relevância para a análise da aderência dos requisitos iniciais aos resultados obtidos. Os SCI são, além de complexos, submetidos a situações extremas em sua operação. As economias e ganhos em segurança resultantes de um planejamento rígido do projeto do SCI em TAs justificam os esforços direcionados para uma maior compreensão e controle da dinâmica dos SCI.

Relevante destacar que na revisão bibliográfica que deu suporte a este trabalho, assim como em buscas feitas na internet, não foi encontrado nenhum trabalho que utilizasse as redes de Petri coloridas para a modelagem de SCI em terminais aquaviários. Sendo assim, pode-se constatar que o presente trabalho é relativamente original, constituindo-se como uma pequena contribuição científica para a área de Planejamento e Projeto de Sistemas Instrumentados de Segurança.

Enquanto as modelagens correntes de simulação consideram as variáveis do sistema de uma forma agregada, o emprego das redes de Petri coloridas permite o acompanhamento de diversas variáveis, tais como estado dos equipamentos, tempo de ativação de cada recurso e diferenciação dos equipamentos, de uma forma simples e clara.

$\mathrm{O}$ formalismo matemático característico à técnica supracitada, também é um diferencial no modelo desenvolvido neste trabalho, no tocante à utilização de outras metodologias de modelagem. A análise de propriedades estruturais e comportamentais pode propiciar ao analista uma maior compreensão da realidade modelada.

O software CPNTools, editor de redes de Petri coloridas utilizado neste trabalho, possui uma interface orientada a objetos bastante amigável e intuitiva para usuários de microcomputadores. Tal fato corrobora o emprego do modelo proposto no planejamento e projeto de sistemas industriais diversos. Para que tal utilização se concretize, torna-se necessário a realização de treinamento do corpo técnico do TA para que este possa realizar, com autonomia, simulações de modo a subsidiar a tomada de decisão em projetos ou na análise dos sistemas industriais do terminal.

\section{AGRADECIMENTOS}

Ao Instituto de Tecnologia e Educação Galileo da Amazônia (ITEGAM) e a Universidade Federal do Pará (UFPA).

\section{REFERÊNCIAS}

[1] NFPA., 1997, Fire Protection Handbook, 16th edition. Quincy, Massachusetts.

[2] Arata, W., 2005, Representação computacional de sistemas a eventos discretos considerando a heterogeneidade e a integração dos modelos. São Paulo, 2005. 188f. Tese (Doutorado) - Escola Politécnica, Universidade de São Paulo, São Paulo.

[3] Seila, A., 1995, Introduction to simulation. In: 27th Winter Simulation Conference. Arlington, 1995. Proceeding. New York.

[4] Miyagi, P.E., 1996, Controle programável -fundamentos do controle de sistemas a eventos discretos, Editora Edgard Blucher, São Paulo. 7.

[5] Ramadge, P.J.; Wonham, W.M., 1989, The control of discrete event systems. In: IEEE. Proceedings. v.77, n. 1.

[6] Villani, E; Kaneshiro, P.I.; Miyagi, P.E., 2005, Uncertainty in Hybrid Systems and the Fire Management System Design. In: 16th IFAC World Congress, 2005, Prague.

[7] Villani, E., 2004, Modelagem e análise de sistemas supervisórios híbridos. São Paulo. 339f. Tese (Doutorado) Escola Politécnica, Universidade de São Paulo, São Paulo.

[8] Silva, M., 1985, Las Redes de Petri: en la Automática y la Informática. Madrid: Editorial AC.

[9] Cardoso, J. E; Valette, R., 1997, Redes de Petri. Florianópolis: Editora da UFSC. 220p.

[10] Peterson, J. L., 1981, Petri Net Theory and the Modelling of Systems. Prentice Hall Inc, New Jersey.

[11] Reisig, W. A Primer in Petri Design. Berlin: SpringerVerlag, 1992.

[12] Hasegawa, K.; Miyagi, P.E..; Takahashi, K., 1989, Mark Flow Graph (MFG) para modelamento e controle de sistemas de eventos discretos. Monografias em Automação e Inteligência Artificial. São Paulo. v.1, n.1, p.1-10.

[13] Murata, T., 1989, Petri nets: properties, analysis and applications. Proceedings of the IEEE, New York, v. 77, n. 4, pp. $541-580$ 
[14]. J.O. Moody, P.J. Antsaklis, Supervisory Control of Discrete Event Systems Using Petri Nets, Kluwer Academic Publishers, Boston, 1998.

[15] M. Silva, Introducing Petri nets, in: F. Di Cesare (Ed.), Practice of Petri Nets in Manufacturing, Chapman \& Hall, 1993, pp. 1-62.

[16] G. Balbo, M. Silva (Eds.), Performance Models for Discrete Event Systems with Synchronizations: Formalisms and Analysis Techniques, Editorial Kronos, Zaragoza, Spain, 1998.

[17] J.I. Latorre-Biel, E. Jiménez-Macías, M. Pérez, The exclusive entities in the formalization of a decision problem based on a discrete event system by means of Petri nets, in: Proceedings of the 23rd European Modelling and Simulation Symposium (EMSS 11), Rome, Italy, 2011, pp. 580-586.

[18] J.I. Latorre, E. Jiménez, M. Pérez, Simulation-based optimization for the designof discrete event systems modeled by parametric Petri nets, in: Proceedings of the UKSim 5th European Modelling Symposium on Mathematical Modelling and Computer Simulation (EMS2011), Madrid, 2011, pp. 150-155.

[19] L.G. Maciel, I.F.Benitez.P, Proposal of Smart Grids in communities of Amazonas State, ITEGAM-JETIA Vol.01, No 02, Manaus, Brazil, 2015, pp.76-82.

[20] Reisig, W., 1985, Petri Nets an Introduction. Springer Verlag, New York.

[21] Jensen, K., 1992, Coloured Petri nets: basic concepts, analysis methods and practical use - Volume 1: basic concepts. Springler-Verlag. Denmark.

[22] Barros, J.P.M.P., 2001, Introdução à modelação de sistemas utilizando redes de Petri. Instituto Politécnico de Beja. Escola Superior de Tecnologia e Gestão. Portugal.

[23] Desrochers, A.A. e Al-Jaar, R.Y., 1995, Applications of Petri nets in manufacturing systems. IEEE Press. United States of America.

[24] Petrobras, N-1203 - Projeto de Sistemas Fixos De Proteção Contra Incêndio em Instalações Terrestres com Hidrocarbonetos. Norma Interna, Rio de Janeiro, 2007. 